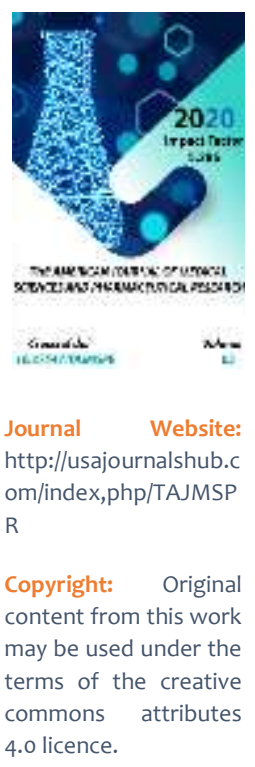

\title{
Hypocalcaemia Among Patients With Psoriatic Disease; A Cross-Sectional Study Among Patients From BSMMU
}

\author{
Emily Akter \\ Assistant Professor, Department Of Dermatology And Venerology, Enam Medical College And \\ Hospital, Dhaka, Bangladesh \\ Lubna Khondker \\ Associate Professor, Department Of Dermatology And Venerology, Bangabandhu Sheikh \\ Mujib Medical University (BSMMU), Dhaka, Bangladesh \\ Mohammad Farhadul Haque \\ Medical Officer, Directorate General Of Health Services (DGHS), Dhaka, Bangladesh \\ Farah Safa Huq \\ Medical Officer, 250 Bedded Mohammad Ali Hospital, Bogra, Bangladesh \\ Md Mahmudul Hasan* \\ Coordinator, Inclusive Health And Education, BMBF, Dhaka, Bangladesh \\ *Corresponding Author: Md Mahmudul Hasan, Coordinator, Inclusive Health And \\ Education, BMBF, Dhaka, Bangladesh
}

\section{ABSTRACT}

Psoriasis is a common, chronic, immune mediated inflammatory disease that involves the innate immunological system and acquired immunological system. Intracellular calcium plays an important part in the regulation of proliferation and differentiation of keratinocytes. This study tried to identify the correlation between severity of disease and serum calcium level in psoriasis patients. To assess the serum calcium level in patients of different types of psoriasis and to find out its relationship with disease severity according to Psoriasis Area Severity Index (PASI).This cross sectional study was conducted among purposively selected 45 patients in the Department of Dermatology and Venereology, Bangabandhu Sheikh Mujib Medical University (BSMMU), Dhaka who had psoriasis on the basis of history, clinical findings and laboratory report. Regardless of gender, the age ranging from 18-65 years and having no calcium metabolism disorder or impaired renal function were the selection criteria of study participants. All patients were interviewed by Pre-structured questionnaire. Blood sample $(5 \mathrm{ml})$ was collected from each subject and was preserved and analyzed for serum calcium level by the automated analyzer i.e. Beckman Coulter-AU680 in the Department of Biochemistry, BSMMU. Age range of the participants were 18 to 65 years (mean age $=34.1 \pm 12.1$ years). Maximum 26 (57.8\%) patients had normal calcium level, 19 (42.2\%) patients had hypocalcaemia and no patient was found with hypercalcaemia. As observed, hypocalcemia frequency is high in more severe types of psoriasis. Hypocalcaemia was found among all types of psoriatic patients'. The correlation between PASI and 
serum calcium levels were moderate negative and significant $(r=-0.558, p<0.001)$. Present study revealed negative correlation between disease severity of psoriasis with patient's serum calcium level. So, serum calcium level can be considered as a marker for disease activity.

\section{KEYWORDS}

Psoriasis, Psoriasis Area Severity Index (PASI), Serum Calcium Level, Serum Albumin Level.

\section{INTRODUCTION}

Psoriasis is a chronic, noncontagious T-cell mediated inflammatory disease with a global prevalence of $2-3 \%$ [1] . In most developed countries, prevalence is between 1.5 to $5 \%$ [2] . There is also evidence to suggest that the prevalence of psoriasis may be increasing [3]. Psoriasis can occur at any age [4] involving the skin and nails, and is associated with a number of comorbidities. Skin lesions can be either localized or generalized, mostly symmetrical, sharply demarcated, have red papules and plaques, and usually covered with white or silver scales. Joint deformities and disability from chronic inflammatory arthritis due to psoriasis was found among $1.3 \%$ in 1995 [5] and $34.7 \%$ [6] of individuals in 2015. Between $4.2 \%$ and $69 \%$ of all patients sufferings from psoriasis develop nail changes [7]. A number of risk factors have been recognized in the etiology and pathogenesis of psoriasis, including family history and environmental risk factors, such as diet, obesity, smoking and alcohol consumption [8]. Psychological factors can trigger the onset or exacerbation of disease [9] - Drug intake was found to be a triggering factor among $36 \%$ of patients. Family records and some Human Leukocytes Antigens (HLA) have also been identified as triggering factor for psoriasis [10].
The autoimmune pathogenesis of psoriasis depends on genetic, environmental and hormonal factors along with factors like immune defects [11]. Environmental factors stimulate cytokines secretion by T-cells that lead to keratinocytes proliferation. In dermal blood vessels, it will also lead to antigenic adhesion molecules production [12].

Psoriatic skin lesions are characterized by a thickened epidermis due to increased keratinocyte proliferation, abnormal differentiation and the infiltration of inflammatory cells into the dermis and epidermis [13] and intracellular calcium plays a central role in regulating this proliferation and differentiation of keratinocytes [14] . A calcium gradient within the epidermis promotes the sequential differentiation of keratinocytes as they traverse the different layers of the epidermis to form the permeability barrier of the stratum corneum. Calcium promotes differentiation by both outside-in and insideout signaling. In the epidermis, calcium gradients from low levels in the proliferative basal layer to high levels in the differentiated granular layer, have been reported. This gradient disappears after psoriasis is developed [15]. About $45 \%$ of serum calcium connects to albumin, therefore, serum calcium, 
especially ionized calcium level depends on serum albumin level and it is better to measure serum albumin level while measuring serum calcium level. Under hypoalbuminemia conditions, the measured number should be corrected in order to obtain real numbers [16] . It has been demonstrated that hypocalcemia may lead to intensifying and extending the lesions in most patients and may lead to development of generalized pustular psoriasis [17]. Altered systemic calcium metabolism has also been observed in various forms of skin lesions [18].

Total serum calcium alterations in psoriasis patients have been varyingly reported [18]. Alteration in systemic calcium level may play a role in the formation of psoriatic skin lesions. This study was designed to assess serum calcium levels in different etiological types of psoriasis. Based on these findings, calcium supplements would be prescribed to psoriatic patients if needed, thus resulting in early recognition of the triggering factors, leading to prevention of disease exacerbation and better management. As there is lack of data regarding serum calcium levels of psoriasis patients among Bangladeshi population, this article aims to estimate the serum calcium level in psoriasis patients and to find out its relationship with disease severity according to Psoriasis Area Severity Index (PASI) among Bangladeshi population.

\section{METHODOLOGY}

This cross sectional study was conducted in the Department of Dermatology \& Venereology at Bangabandhu Sheikh Mujib Medical University (BSMMU), Dhaka and was completed in oneyear period between 1st September 2017 to 31th August 2018. Newly diagnosed psoriasis individuals were the study subjects. In order to collect the sample, purposive sampling technique was applied. Individuals were screened on the basis of selection criteria of the study. Individuals aged between 18 to 65 years regardless of gender were selected. Patients having calcium metabolism disorder, bone metabolism disorder, impaired renal functions, pre-existing renal diseases, on drugs causing calcium metabolism disorder (Lithium, Thiazide Diuretics, Thyroxine, Calcium Carbonate, Vitamin D) or on concomitant use of hepatotoxic or nephrotoxic drugs for any other long-standing illness were excluded from study. Subjects who were treated for psoriasis in the past were also excluded from the study. A prior study was used to set up and calculate the sample size of 45 [19].

Data was collected from the eligible participants after getting informed consent from them. Information about age, gender, present illness, and duration of the disease, comorbidities and drug history was taken from the participants through a pre-structured questionnaire . A thorough dermatological examination was done to ascertain the extent of the disease. The severity of disease was measured by using severity assessment tools namely, Psoriasis Area and Severity Index (PASI). A PASI score $>12$ is considered as severe psoriasis. If it is in between 7-12 it is considered as moderate and PASI $<7$ is regarded as mild form of psoriasis [20]. Blood sample ( $5 \mathrm{ml})$ was collected from each patient, and these were preserved and analyzed for serum calcium and albumin levels by the use of o-cresolphthalein complexone (OCPC)/8-quinolinol and glycine buffer in the automated analyzer: Beckman Coulter-AU680 in the Department of Biochemistry, BSMMU. The expected values for serum calcium is $8.5-10.5 \mathrm{mg} / \mathrm{dl}$ and the expected values for serum albumin is $35-50$ g/L. 
Prior to implement the main procedure, a pretest was done by taking 5 samples among the study population. Collected data was verified and cross-checked. A quality control sample (QC) and a fixed standard was used in every assay run for each of the variables for assessment over coefficient variance (CV) and precision of the study. All the relevant collected data were compiled. Statistical analyses were carried out by using Statistical Package for Social Sciences version 25.0 for Windows. Necessary ethical clearance for the study was sought from the Institutional Review Board and concerned authority, BSMMU.

\section{RESULTS}

\begin{tabular}{|lcccccc|}
\hline & \multicolumn{2}{c}{ Male } & \multicolumn{2}{c}{ Female } & \multicolumn{2}{c|}{ Total } \\
\cline { 2 - 7 } & $\mathrm{n}$ & $\%$ & $\mathrm{n}$ & $\%$ & $\mathrm{n}$ & $\%$ \\
\hline Age Group & & & & & & \\
\hline $18-29$ years & $19 / 23$ & 82.6 & $4 / 23$ & 17.4 & $23 / 45$ & 51.1 \\
\hline $30-44$ years & $10 / 13$ & 76.9 & $3 / 13$ & 23.1 & $13 / 45$ & 28.9 \\
\hline $45-59$ years & $4 / 7$ & 57.1 & $3 / 7$ & 42.9 & $7 / 45$ & 15.6 \\
\hline 60 and above & $2 / 2$ & 100.0 & $0 / 2$ & 0.0 & $2 / 45$ & 4.4 \\
\hline Types of psoriasis & & & & & & \\
\hline Vulgaris psoriasis & $19 / 23$ & 82.6 & $4 / 23$ & 17.4 & $23 / 45$ & 51.1 \\
\hline Dispersed pustular psoriasis & $9 / 12$ & 75.0 & $3 / 12$ & 25.0 & $12 / 45$ & 26.7 \\
\hline Erythrodermic psoriasis & $4 / 6$ & 66.7 & $2 / 6$ & 33.3 & $6 / 45$ & 13.3 \\
\hline Vulgaris psoriasis along with & $3 / 4$ & 75.0 & $1 / 4$ & 25.0 & $4 / 45$ & 8.9 \\
psoriatic arthritis & & & & & & \\
\hline Severity of Psoriasis (by PASI) & & & & & & \\
\hline Mild Disease (PASI <7) & $6 / 6$ & 100.0 & 0 & 0 & $6 / 45$ & 13.3 \\
\hline Moderate Disease (PASI 7-12) & $15 / 17$ & 88.2 & $2 / 17$ & 11.8 & $17 / 45$ & 37.8 \\
\hline Severe Disease (PASI >12) & $14 / 22$ & 63.6 & $8 / 22$ & 36.4 & $22 / 45$ & 48.9 \\
\hline Total & $35 / 45$ & 77.8 & $10 / 45$ & 22.2 & 45 & 100.0 \\
\hline
\end{tabular}

Table 1: Distribution of study population according to Age group, Gender, Types and Severity of Psoriasis. $(\mathrm{N}=45)$

There were $77.8 \%$ male and $22.2 \%$ female among the participants. Also, $51.1 \%$ of study population was from age group 18 -29 years followed by a steady decline at subsequent age groups (Table 1). When study population was distributed according to the types of psoriasis, $51.1 \%$ of the study population were found to be suffering from Vulgaris Psoriasis (male: female=82.6\%: 17.4\%). Dispersed Pustular
Psoriasis was found among $26.7 \%$ of the study population (75.0\% were male and $25.0 \%$ were female) while Erythrodermic Psoriasis was found among $13.3 \%$ of the study population (male : female $=66.7 \%: 33.3 \%$ ) and Vulgaris Psoriasis along with Psoriatic Arthritis was found among $8.9 \%$ of the study population where male and female was $75 \%$ and $25 \%$ respectively (Table 1) 
PASI score was used for measuring the disease severity. Mild disease (PASI <7) was found among $13.3 \%$ of the population and consisted entirely of the male population. On the other hand, moderate disease (PASI 7-12) and severe disease (PASI >12) was found among $37.8 \%$ ( $88.2 \%$ were male and $11.8 \%$ were female) and $48.9 \%$ (63.6\% were male and $36.4 \%$ were female) of the study population respectively (Table 1).

\begin{tabular}{|lcccccc|}
\hline & \multicolumn{2}{c}{ Male } & \multicolumn{2}{c}{ Female } & \multicolumn{2}{c|}{ Total } \\
\cline { 2 - 7 } & $\mathrm{n}$ & $\%$ & $\mathrm{n}$ & $\%$ & $\mathrm{n}$ & $\%$ \\
\hline Serum Calcium Level & & & & & & \\
\hline Hypocalcemia $(<8.5 \mathrm{mg} / \mathrm{dl})$ & $15 / 19$ & 78.9 & $4 / 19$ & 21.1 & $19 / 45$ & 42.2 \\
\hline Normal $(8.5-10.5 \mathrm{mg} / \mathrm{dl})$ & $20 / 26$ & 76.9 & $6 / 26$ & 23.1 & $26 / 45$ & 57.8 \\
\hline Hypercalcemia $(>10.5 \mathrm{mg} / \mathrm{dl})$ & 0 & 0 & 0 & 0 & 0 & 0 \\
\hline Serum Albumin Level & & & & & & \\
\hline Hypoalbuminemia $(<35 \mathrm{gm} / \mathrm{L})$ & $30 / 40$ & 75.0 & $10 / 40$ & 25.0 & $40 / 45$ & 88.9 \\
\hline Normal $(35-50$ gm/L) & $5 / 5$ & 100.0 & 0 & 0 & $5 / 45$ & 11.1 \\
\hline Hyperalbuminemia $(>50 \mathrm{gm} / \mathrm{L})$ & 0 & 0 & 0 & 0 & 0 & 0 \\
\hline Total & $35 / 45$ & 77.8 & $10 / 45$ & 22.2 & 45 & 100.0 \\
\hline
\end{tabular}

Table 2: Distribution of study population according to serum Calcium level and Serum Albumin Level. $(\mathrm{N}=45)$

Serum calcium levels of the study population were measured, and $42.2 \%$ were found to be suffering from Hypocalcaemia and $57.8 \%$ had normal serum calcium level (Table 2). Serum albumin levels were also estimated and $88.9 \%$ were found to have been suffering from Hypoalbuminemia and $11.1 \%$ had normal serum albumin level (Table 2). Hypercalcemia or Hyperalbuminemia was absent among any of the study samples.

\begin{tabular}{|lccc|}
\hline Severity of Psoriasis (by PASI) & $\begin{array}{c}\text { Frequency } \\
\text { (Percentage) }\end{array}$ & $\begin{array}{c}\text { Mean S. Calcium Levels } \\
(\mathrm{mg} / \mathrm{dl})\end{array}$ & ANOVA \\
\cline { 1 - 3 } Mild Disease (PASI <7) & $6(13.3 \%)$ & 10.13 & $\mathrm{P}<0.001$ \\
\cline { 1 - 3 } Moderate Disease (PASI 7-12) & $17(37.8 \%)$ & 8.88 & \\
\hline Severe Disease (PASI >12) & $22(48.9 \%)$ & 8.04 & \\
\hline
\end{tabular}

Table 3: Comparison of Serum Calcium Level with Severity of Psoriasis (by PASI). $(\mathrm{N}=45)$

Disease severity was measured using Psoriasis Area and Severity Index (PASI) (Table 3). Mild disease (PASI $<7$ ) was noticed among $13.3 \%$ of the study population, where moderate disease (PASI 7-12) was observed among $37.8 \%$ of the study population. About $48.9 \%$ of the study 
population had showed severe disease (PASI >

12). The difference in mean serum calcium levels among groups was found to be statistically significant $(p<0.001)$ (Table 3$)$.

\begin{tabular}{|c|c|c|c|}
\hline Severity of Psoriasis (by PASI) & $\begin{array}{c}\text { Hypocalcemia } \\
(<8.5 \mathrm{mg} / \mathrm{dl})\end{array}$ & $\begin{array}{c}\text { Normal Serum } \\
\text { Calcium Level }(8.5-10.5 \\
\mathrm{mg} / \mathrm{dl})\end{array}$ & Significance \\
\hline Mild Disease (PASI <7) & $0(0 \%)$ & $6(100.0 \%)$ & \multirow{3}{*}{$\mathrm{p}<0.001$} \\
\hline Moderate Disease (PASI 7-12) & $8(47.1 \%)$ & $9(52.9 \%)$ & \\
\hline Severe Disease (PASI >12) & $18(81.8 \%)$ & $4(18.2 \%)$ & \\
\hline
\end{tabular}

Table 4: Association between Serum Calcium Level and Severity of Psoriasis (by PASI). ( $\mathrm{N}=$

45)

All of the respondents suffering from mild disease had normal serum calcium level (8.5$10.5 \mathrm{mg} / \mathrm{dl}$ ), where $47.1 \%$ of the respondents with moderate disease developed hypocalcemia (<8.5 mg/dl) (Table 4). The prevalence of hypocalcaemia was found to be

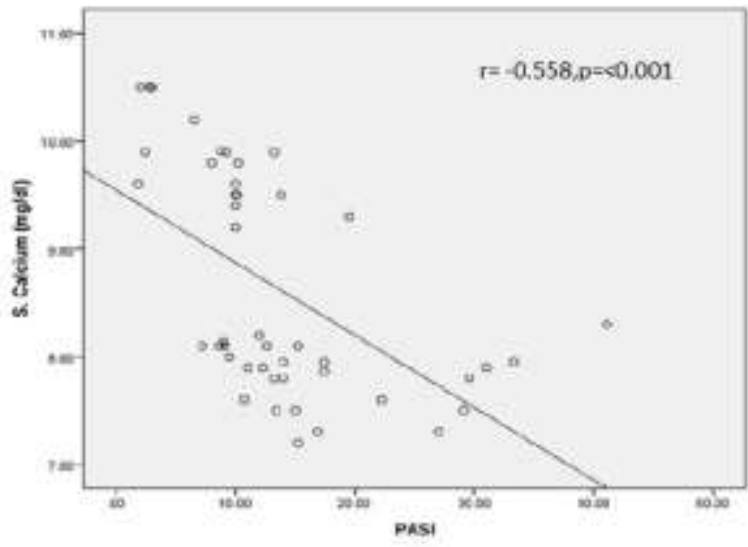

A increasing with the severity of the disease. Among the respondents suffering from the severe disease, $81.8 \%$ had developed hypocalcaemia. Statistically significant ( $p<$ 0.001 ) association was found between severity of psoriasis and serum calcium level (Table 4).

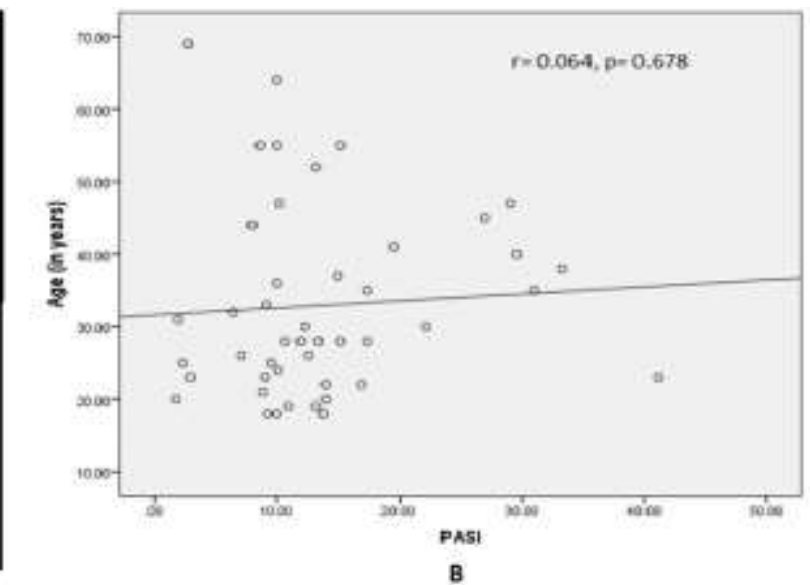

Figure 1: A- Correlation of PASI with serum calcium level \& B-Correlation of PASI with age of the study population 
It is noticed that the PASI has negative correlation with Serum calcium level as the $r=$ 0.558 and $p=<0.001$ (Figure 1: A) and a weak positive correlation with the age because it shows $r=0.064$ and $p=0.678$ (Figure 1 : $B$ ).

\section{DISCUSSION}

This cross-sectional study was carried out with the aim to measure the serum calcium level in psoriatic patients and to find out its relationship with disease severity. In the present study, the age range of the patient was 18 to 65 years. The mean age of patients was $34.1 \pm 12.1$ years and maximum $51.1 \%$ of patients were $21-30$ years of age and $24.4 \%$ of patients were 31-40 years of age which means about $75.5 \%$ of patients were between 21 and 40 years of age. This finding is quite similar with the prior studies which showed dual peaks for psoriasis located in the $20 \mathrm{~s}$ and $40 \mathrm{~s}[21,22]$. $33 \pm 13.02$ years were found as the mean age in previous research [23] which is consistent with the present study. In regards to the gender dominance of the disease, this study shows a higher percentage of man than woman. Similarly, studies had shown that the incidence of this disease is more noticeable in men than in women [24, 23]. In contrast, the male and female ratio of this disease is considered to be 1:1 globally [25]. In Bangladesh, women seek less medical attention for their illness due to cultural, social \& religious aspects \& may be that could be the reason of male predominance.

Psoriasis is categorized as hyper proliferative disease, as epidermal basal cells exhibit increased frequency of mitosis in the disease. Several studies have demonstrated close relation between psoriasis and serum calcium level [24] . In present study out of 45 patients, 19 (42.2\%) patients had hypocalcaemia which correlated with a study done by Qadim et al. (2013) [19]. Puri and Mahajan (2016) conducted a study where before the start of the treatment, hypocalcaemia was seen in 10\% patients and at the end of treatment, serum calcium levels were normal in all the patients $(p<0.05)$ [26]. According to the results of the present study, low level of serum calcium was observed in almost all patients of erythrodermic and half of the patients suffering from pustular psoriasis. The results are in correspondence with other research [27].

In regards to the albumin, the present study showed that about $88.9 \%$ of patients had normal albumin. Unlike this finding, the serum albumin level was found as low among the majority (64.9\%) of hypocalcaemia psoriatic patients in a case control study [19]. The comparison of value of serum calcium level among different severity of psoriasis cases showed significant difference among mild, moderate and severe form of psoriasis ( $p<$ 0.05). This finding is consistent with a prior study conducted by Chaudhari and Rathi (2018) [28]. In addition, the present study showed the relationship between hypocalcaemia with different types of psoriasis $(p<0.05)$. Similarly, calcium serum levels were compared considering types of psoriasis in the study conducted by Qadim et al. (2013) [19].

In present study, almost $81.8 \%$ of patients with hypocalcaemia show the PASI score of more than 12 where $47.1 \%$ of patients with hypocalcaemia show a moderate PASI score of 7-12. Interestingly, patients with mild PASI score (>7) did not show hypocalcaemia in this study. In contrast, Bijina et al. (2018) study found hypocalcaemia in all PASI scores from 0 to 45 [24]. The use of non-unique measurement scales in studies may be responsible for the display of these different results. Therefore, a 
universal measure scale in this regard is suggested.

Present study depicts the correlation of PASI with the age of patient and serum calcium level. Correlation coefficient in the serum calcium was -0.558 whereas in age was 0.064 . Serum calcium level had significant moderate negative correlation with PASI and age had insignificant weak positive correlation with PASI. A study done by Bijina et al. (2018) reported negative correlation between hypocalcaemia and PASI score [24]. P value of that study was 0.001 , which was significant and consistent with the findings of present study.

\section{Limitation}

The study was conducted using only 45 samples. Having such a small sample size and the use of the purposive sampling technique had made it difficult to account for selection bias and conduct in-depth statistical analysis. Conducting cross-sectional studies also limited our scope for data collection and, as a result, no follow-up was performed on any of the subsequent dates by researchers to assess changes in serum calcium levels over time as the disease progressed.

\section{CONCLUSION \& RECOMIMENDATION}

This study, conducted among 45 psoriatic patients of Bangladesh, found significant negative correlation between PASI score and serum calcium level. In view of the result from both present study and other studies mentioned, it can be concluded that serum calcium level may add a valuable insight for the physicians determining the severity of the disease. Serum calcium level should be routinely measured in patients with psoriasis, especially in those with more severe disease. Further study should be conducted with much larger sample size to increase the credibility and validity of these findings. In addtion, conducting a multi-center cohort study to evaluate the serum calcium levels in participants over an extended period of time will give us better understanding on the intricate relationship between serum calcium levels and the severity of the disease among psoriasis patients.

\section{REFERENCES}

1. National Psoriasis Foundation. About Psoriasis. Statistics. Available at: http://www.psoriasis.org/about/stats/ [accessed 23 March 2015].

2. Parisi R, Symmons DPM, Griffiths CEM, Ashcroft DM, . Identification and management of psoriasis and associated comorbidity (IMPACT) project team, 2013, Global epidemiology of psoriasis: a systematic review of incidence and prevalence, J Invest Dermatol 2013;133:377-85

3. Danielsen $\mathrm{K}$, Olsen $\mathrm{AO}$, Wilsgaard $\mathrm{T}$, Furberg AS,. Is the prevalence of psoriasis increasing? A 30-year followup of a population-based cohort 2013; 168:1303-10.

4. Institute for Health Metrics and Evaluation (IHME), . Global Burden of Disease Study 2010: Results by Cause 1990-2010, 2012; Seattle: IHME.

5. Bedi TR, . Clinical profile of psoriasis in North India. Indian J Dermatol Venereol Leprol 1995; 61: 202-5.

6. Pariser D, Schenkel B, Carter C, Farahi K, Brown TM, . Patient Interview Study Group. A multicenter, noninterventional study to evaluate patient-reported experiences of living with psoriasis, J Dermatol Treat 2015; 18. 
7. Falodun OA, Characteristics of patients with psoriasis seen at the dermatology clinic of a tertiary hospital in Nigeria: a 4-year review 2008-2012. J Eur Acad Dermatol Venereol 2013;27.

8. Huerta C, RivZero E, Rodriguez LA, Incidence and risk factors for psoriasis in the general population. Arch Dermatol 2007; 143(12); 1559-65.

9. Fortune DG, Main CJ, O'Sullivan TM, Griffiths CE, . Quality of life in patients with psoriasis: the contribution of clinical variables and psoriasis specific stress', Br J Dermatol 1997;137:755-60.

10. Duweb G, Alhaddar J, Abuhamida M,. Psoriasis vulgaris: once-versus twicedaily application of calcipotriol cream. Int J Tissue React 2005;27:155-8.

11. Ozawa $M$, Aiba S,. Immunopathogenesis of psoriasis. Current Drug Targets: Inflammation \& Allergy 2004; 3:137-144.

12. Wisnieski JJ ,. Urticarial vasculitis. Current Opinion in Rheumatilogy 200; 12:24-31.

13. Griffiths CE, Barker JN,. Pathogenesis and clinical features of psoriasis. Lancet 2007; 370:263-271.

14. Bikle DD, Xie Z, Tu CL,. Calcium regulation of keratinocyte differentiation. Expert Review of Endocrinology \& Metabolism 2012; 7:461-72.

15. Cubillos S, Norgauer J,. Low vitamin Dmodulated calcium-regulating proteins in psoriasis vulgaris plaques: $\mathrm{S} 100 \mathrm{~A} 7$ overexpression depends on joint involvement. International Journal of Molecular Medicine 2016; 38:1083-92.

16. Matsushita $\mathrm{Y}$, Shimada $\mathrm{Y}$, Kawara $\mathrm{S}$, Takehara K, Sato K,. Autoantibodies directed against the protease inhibitor calpastatin in psoriasis . Clin. Exp. Immunol 2005;39:355-62

17. Lee Y, Nam YH, Lee IH, Park JK, Seo YJ,. Hypocalcaemia induced pustular psoriasis like skin eruption.Br. I. Dermatol 2005:152:591-93.

18. Plavina $T$, Hincapie $M$, Wakshull $E$, Subramanyam M, Hancock WS,. Increased plasma concentrations of cytoskeletal and Ca2tbinding proteins and their peptides in psoriasis patients. Clin Chem 2008; 54:1805-14.

19. Qadim HH, Goforoushan F, Nejad SB, Goldust $M$,.Studying the calcium serum level in patients suffering from psoriasis. Pak. J. Biol. Sci 2013; 16:291294.

20. Schmitt J, Wozel G,. The psoriasis area and severity index is the adequate criterion to define severity in chronic plaque-type psoriasis. Dermatology 2005; 210:194-99.

21. Kawada A, Tezuka T, Nakamizo Y, Kimura $\mathrm{H}$, Nakagawa $\mathrm{H}$, Ohkido $\mathrm{M}$, et al, . A survey of psoriasis patients in Japan from 1982 to 2001. J Dermatol Sci 2003;31:59-64.

22. Ding $X$, Wang $T$, Shen $Y$, Wang $X$, Zhou $C$, et al,.Prevalence of psoriasis in China: a population-based study in six cities. Eur J Dermatol. 2012;22: 663-7.

23. Keen MA, Hassan I,. Serum prolactin levels in psoriasis and its association with disease activity: a case-control study. Indian journal of dermatology 2014; 59:562-66.

24. Bijina KD, Raghavendra BN, Mohamed $M,$. A study of serum calcium and uric acid levels in Psoriasis. Indian J Clin Exp Dermatol 2018; 4:342-45.

25. World Health Organization,. Global report on psoriasis. Available at: 
https://apps.who.int/iris/handle/10665/ 204417 [accessed: 1 April 2015]

26. Puri N, Mahajan BB,. A study of clinical and biochemical correlation in patients of psoriasis in acute exacerbation. Journal of Pakistan Association of Dermatology 2016;24:236-40.

27. Kitamura K, Kanasashi M, Suga C, Saito S, Yoshida S, Ikezawa Z,. Cutaneous reactions induced by calcium channel blocker: high frequency of psoriasiform eruptions. J Dermatol 1993;20:279-86.

28. Chaudhari S, Rathi S,. Correlation of serum calcium levels with severity of psoriasis. Int J Res Dermatol 2018;

4:591-4 